\title{
25-mal nachhaltige Zukunft für Städte und Gemeinden
}

\begin{abstract}
Städte sind wichtigster Lebensraum der Erde und umweltbelastendste Organisationen in einem. Zur Lösung dieses Dilemmas finden unterschiedliche Instrumente ihre Anwendung. Ein europäisches Projekt begleitet nun Städte und Gemeinden auf dem Weg zur Einführung eines integrierten Nachhaltigkeitsmanagements. Von Holger Robrecht
\end{abstract}

$D^{2}$ er maßgebende 'Best Insurance Underwriting Guide', den Risikoexperten US-amerikanischer Versicherungsunternehmen zur Einschätzung geschäftlicher Risiken nutzen, listet Kommunen als die am meisten umweltgefährdenden Organisationen, noch vor der Petrochemischen Industrie. Wir dürfen getrost annehmen, dass ähnliche Einschätzungen auch in Europa zutreffen. Und das trotz enormer Umweltleistungen, die Kommunen in der Vergangenheit erbracht haben. Und trotz einer immensen Regelungsdichte auf europäischer wie nationaler Ebene. Große Herausforderungen bleiben bestehen, die Lebensqualität in unseren Städten und Gemeinden zu verbessern und für unsere nachfolgenden Generationen aufrechtzuerhalten. Kurz: eine nachhaltige Stadtentwicklung umzusetzen.

25 europäische Städte, Gemeinden und Regionen - darunter 12 deutsche - haben diese Herausforderung angenommen. Sie richten ihre Stadtentwicklung an Prinzipien einer nachhaltigen Entwicklung aus. Zum ersten Mal wollen sie ein umfassendes Nachhaltigkeitsmanagement für ihr gesamtes, städtisches Gebiet einführen, dass so genannte zivilgesellschaftliche Partner wie Bürger, Firmen und Vereine in wichtigen Schritten einbezieht. Dies zu erreichen bedarf einer Reihe von Maßnahmen, die sich auf das übergeordnete Prinzip einer nachhaltigen Stadtentwicklung beziehen. Dabei ist wichtig, den Kern des Managements im Auge zu behalten. Denn es sind nicht Produkte oder Dienstleistungen, die gemanagt werden. Es sind Menschen. Insofern ist die wichtigste Managementregel die Verbesserung und Steuerung der Kommunikation der Beteiligten.

\section{Managing Urban Europe 25}

Das Projekt Managing Urban Europe 25 zielt auf die Entwicklung eines integrierten Managementsystems für städtische Gebiete (1). Es ist eng mit der Erarbeitung der thematischen Strategie für die städtische Umwelt der Europäischen Kommission verknüpft und das vermutlich größte geförderte Projekt zum Nachhaltigkeitsmanagement für Kommunen. Mehrere europäische Städtenetzwerke und Forschungspartner sind involviert.

Die Teilnehmerkommunen haben unterschiedlichste Rahmenbedingungen. Einwohnerzahlen reichen von 1550 Einwohner in der deutschen Gemeinde Ascha bis hin zu den 4,5 Millionen Einwohnern in Norwegens Hauptstadt Oslo. Kreisangehörige Gemeinden machen genauso mit wie selbständige Städte, Kreise und Regionen. Die Aktivitäten der deutschen Städte und Gemeinden werden von ICLEI - Local Governments for Sustainability und der Bodensee-Stiftung betreut. Das Projekt wird noch bis zum Mai 2008 laufen.

\section{Ein Fahrplan für Europa}

Als das Europaparlament vor einiger Zeit die Europäische Kommission aufforderte, eine „Thematische Strategie für die Städtische Umwelt“ (TSU) vorzulegen, stieß dies innerhalb der Kommission zunächst auf wenig Gegenliebe. Doch erkannten die Autoren des ersten Entwurfes im Februar 2004 die Chance, Umweltmanagementsysteme zumindest in großen Städten mit mehr als 100.000 Einwohnern verpflichtend einzuführen.

Umweltmanagementsysteme waren bis dato freiwillig angeboten, mit Vorschußlorbeeren bezüglich ihrer Wirkung überhäuft und blieben in der Realität weit hinter den Vorstellungen einer breiten Anwendung in Städten und Gemeinden zurück. Viele Entscheidungsträger in Kommunen lehnen noch heute Umweltmanagementsysteme wegen anfänglich schwieriger Erfahrungen mit EMAS als ein aufwändiges, kostspieliges und personalintensives Ungetüm ab. Tatsächlich haben sich EMAS und andere Umweltmanagementsysteme wie das von ICLEI in Zusammenarbeit mit Städten und Gemeinden entwickelte ökoBUDGET an die Verwaltungsnotwendigkeiten angepasst und zeitigen beachtliche Ergebnisse in punkto Effizienzsteigerung, Kosteneinsparung und Umweltleistung.

Am 11. Januar 2006 veröffentlichte die Europäische Kommission die endgültige Fassung einer ,Thematischen Strategie für die Städtische Umwelt‘ (2). Nach Konsultationen innerhalb von Experten-Arbeitsgruppen und mit europäischen Städtenetzwerken spricht die Strategie nunmehr von einem integriertem Managementansatz für das städtische Gebiet. Damit wird ein Nachhaltigkeitsmanagement nahegelegt, gleichwohl dem Mandat der Strategie entsprechend von Umweltherausforderungen gesprochen. Im Laufe des Jahres 2006 sollen Leitlinien zur Umsetzung veröffentlicht werden, die im Kern auch auf den Erfahrungen des Projektes Managing Urban Europe 25 beruhen. $\Rightarrow$ 
Zunehmende Bedeutung erfährt der Ansatz zur Entwicklung eines integrierten Managementansatzes noch durch zwei weitere europäische Prozesse. Zum einen die überarbeitete EU Nachhaltigkeitsstrategie (3) sowie die so genannten Aalborg-Verpflichtungen (4). Die Aalborg-Verpflichtungen, inzwischen von fast 400 europäischen Städten und Gemeinden unterzeichnet, schreiben einen Management-Prozess vor, mit dem Ziele einer nachhaltigen Stadtentwicklung in zehn Handlungsfeldern mit insgesamt 50 Schlüsselaktivitäten erreicht werden sollen. Die Unterzeichner verpflichten sich, eine Bestandsaufnahme innerhalb von zwölf Monaten vorzulegen, gemeinsam mit zivilgesellschaftlichen Gruppen innerhalb von 24 Monaten Ziele zu setzen und Fortschrittsberichte alle drei Jahre zu veröffentlichen. Die EU Nachhaltigkeitsstrategie verknüpft nun diesen kommunalen Prozess mit der Umsetzung der europäischen Nachhaltigkeitsziele (5). Den lokalen und regionalen Ebenen und den damit verbundenen Ansätzen wie der Lokalen Agenda 21 kommt darin ein zentrale Rolle zu.

\section{Reise zur nachhaltigen Stadtentwicklung}

Die Notwendigkeit für ein kommunales Nachhaltigkeitsmanagement folgt der Erkenntnis, dass eine ausschließliche Betrachtung einzelner Felder der Stadtentwicklung nicht mehr ausreicht. Schon in den 80er und 90er Jahren wurde deutlich, dass Umweltpolitik integriert zu sehen ist. Die sektoral ausgerichtete Politik und Verwaltung war nicht imstande, die Verknüpfungen innerhalb unserer Umwelt zu erkennen und aufzugreifen. Doch spätestens seit dem Weltgipfel für Nachhaltige Entwicklung in Johannesburg 2002 und den sogenannten MilleniumEntwicklungszielen der Vereinten Nationen wissen wir, dass auch eine integrierte Umweltpolitik nicht reicht (6). Immer deutlicher und kurzwelliger deuten Wetterextreme auf die Phänomene des sich vollziehenden Klimawandels. Sie sind längst nicht mehr „Umweltproblem“.

Nach Aussage von Hartmut Graß1 hat kein Einzelereignis seit dem 2. Weltkrieg in Europa mehr Opfer gefordert, als der so genannte Jahrhundertsommer 2003, als eine Hitzewelle ungekannten Ausmaßes viele tausend Menschen das Leben kostete. Es waren zumeist ältere Menschen, von ihren Familien auf der Fahrt in die Ferien zu den Seen und Stränden in den Städten zurückgelassen. Ein soziales Desaster, das wohl noch größer war als die wirtschaftlichen Folgen verdorrter Ernten, zusammengebrochener Energieversorgung und vieltausendfacher Arbeitsausfälle durch Hitzefolgen.

Nur konsequent gehen nun Städte und Gemeinden den nächsten Schritt. Unter Nutzung der Erfahrungen mit kommunalen

Quelle:
Umweltmanagementsystemen wird ein Umwelt- und Nachhaltigkeitsmanagementsystem für das gesamte Einfluss- und Wirkungsgebiet von Städten und Gemeinden entwickelt und getestet. Dabei wird ausdrücklich nicht an den Gemeindegrenzen Halt gemacht. Das Managementsystem soll auch dort greifen, wo Gemeindegrenzen überschritten werden und der Verantwortungsbereich von Nachbargemeinden anfängt. Vor allem aber soll es eines erreichen: Nachhaltige Entwicklung soll weg von einer thematischen Verankerung und zu einem Entscheidungsprinzip für kommunales Handeln werden.

Ein integriertes Managementsystem darf nicht das kommunale Mangement auf Null setzen. Die Erfahrungen mit kommunalen Umweltmanagementsystemen zeigen, dass die Einführung eines neuen Instrumentariums unbedingt die Vorleistungen, Startbedingungen und Ambitionen der jeweiligen Gemeinde respektieren muss. Bei näherer Betrachtung zeigt sich, dass Städte und Gemeinden schon immer ein umfangreiches Instrumentarium anwenden. Da gibt es Pläne für die Bebauung und die Lärmminderung. Es gibt Förderprogramme für die Renovierung von Altbauten und die Nutzung regenerativer Energien. Es gibt Gebote für die Verwendung von Regenwasser und Verbote zur freihändigen Entsorgung von Hausabfällen. In vielen Fällen fehlt jedoch die Koordinierung dieser Instrumente. Daraus ergeben sich Doppelarbeiten. So besteht unter Umständen kein Gesamtbild der versiegelten Flächen, weil die Daten an vielen verschiedenen Stellen, die jeweils mit einem Teilaspekt befaßt sind, vorgehalten werden. Das fehlende Wissen um diese einzelnen Stellen bewirkt ein Informationsdefizit. Die Räder greifen nicht ineinander. Wie soll der Gemeinderat die Neuversiegelung einer Innenstadtbrache zum Parkplatz dann beurteilen, wenn kein Gesamtbild und damit kein Maßstab vorliegt?

Abbildung 1: Rahmenmodell für Nachhaltigkeitsmanagement

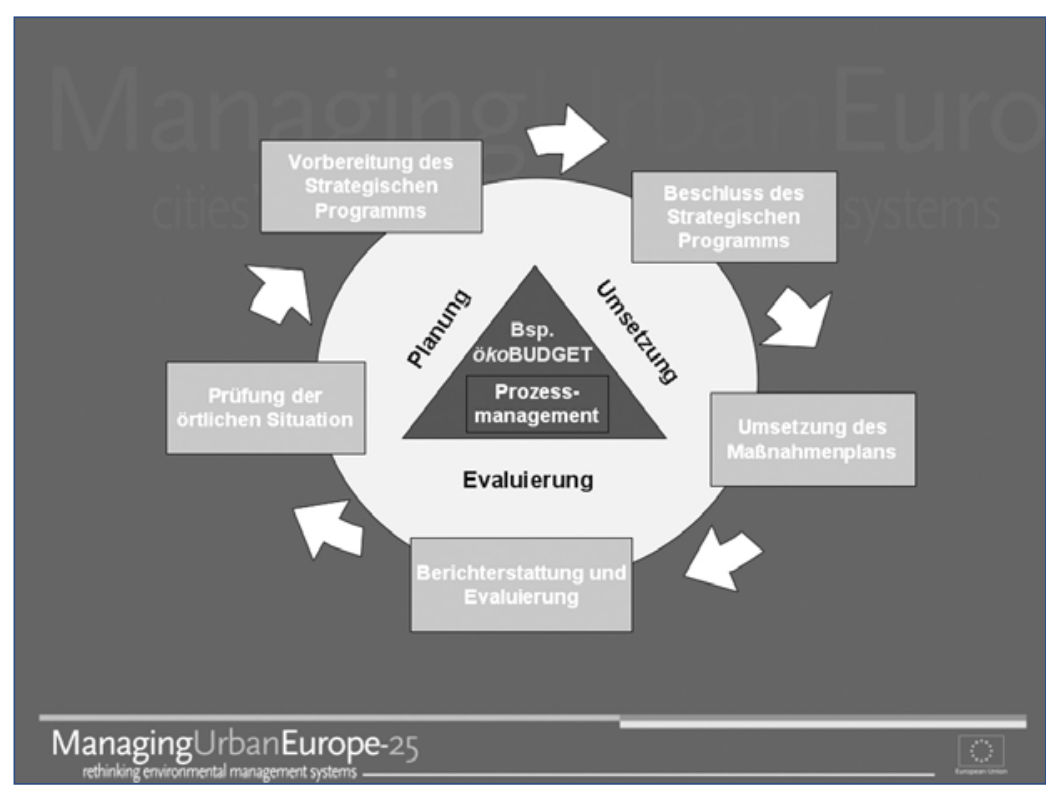

Eigene Darstellung 
Die Projektteilnehmer haben sich auf den Managementzyklus von Planen, Handeln, Beobachten und Reagieren als Grundlage ihrer Arbeiten verständigt. Dieser Zyklus wird um einen für kommunales Management wesentlichen Punkt erweitert: das Entscheiden. Aus den Erfahrungen mit dem Umweltmanagementsystem ökoBUDGET wird deutlich, dass die regelmäßige Einbindung des Gemeinderates ein Schlüssel zum Erfolg und zur Entwicklung eines Prinzips Nachhaltigkeit in städtischen Entscheidungen ist. Damit wird ein Managementrahmen bestehend aus fünf wohlbekannten Elementen gewählt, wie ihn die Abbildung 1 vorstellt. Auf die Entwicklung eines neuen Instrumentes wird verzichtet.

Das Managementsystem fußt auf 13 Schlüsselprinzipien, wie etwa der Relevanz des Systems für die städtischen Akteure, Gesetzmäßigkeit, kontinuierlicher Verbesserung, Verankerung im zentralen Verantwortungsbereich von Verwaltung und Politik, strategischer Orientierung und schrittweiser Erweiterung. Die Einführung des Managementsystems setzt auf den vorhandenen Gegebenheiten auf. Eine Überprüfung der bereits genutzten Instrumente weist auf mögliche Lücken im örtlichen Management hin. Mit einem individuellen Fahrplan werden diese Lücken dann geschlossen und ein koordiniertes Managementsystem installiert. Zugrunde liegt dem die Erfahrung, dass sich Kommunen auf einer mehr oder weniger natürlichen Reise zum Nachhaltigkeitsmanagement befinden. In den unterschiedlichen Phasen der Entwicklung der Umwelt- und Nachhaltigkeitspolitik wurden immer auch entsprechende Instrumente entwickelt und eingeführt. Daraus ergibt sich eine Treppe zur Nachhaltigkeit, wie Abbildung 2 beschreibt.

Im ersten Schritt geht es darum, den Standort der jeweiligen Gemeinde zu bestimmen. Sie wird daraufhin festlegen, wie am-

Abbildung 2: Wo steht Ihre Gemeinde? Die Treppe zum kommunalen Nachhaltigkeitsmanagement

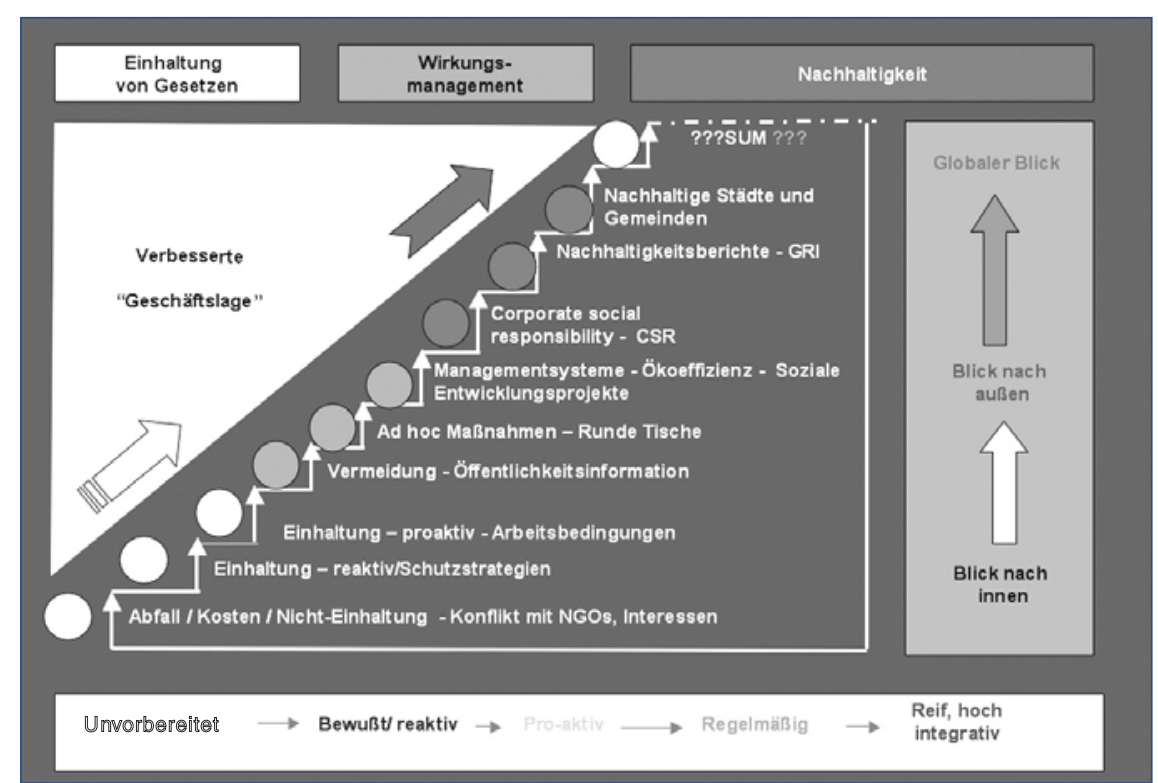

Quelle: Prof. Johan Nel, Center for Environmental Management, North-West University, Potchefstroom, South Africa, 2003
Tabelle 1

\begin{tabular}{|ll}
\hline A1. & Regieren \\
\hline A2. & Kommunales Management für Zukunftsbeständigkeit \\
\hline A3. & Natürliche Gemeinschaftsgüter \\
\hline$A 4$. & Verantwortung in Konsum und Lebensweise \\
\hline A5. & Stadtplanung und -entwicklung \\
\hline A6. & Verbesserte Mobilität, weniger Verkehr \\
\hline A7. & Kommunale, gesundheitsfördernde Maßnahmen \\
\hline A8. & Dynamische und zukunftsbeständige Wirtschaft \\
\hline A9. & Soziale Gerechtigkeit \\
\hline A10. & Globale Verantwortung \\
\hline
\end{tabular}

bitioniert sie auf der Treppe weiterklettern will. Aus dieser Ambition leitet sich dann der Fahrplan ab. Dabei wird kein bestimmter Managementstandard verfolgt. Gleichwohl werden Teilnehmergemeinden zum Abschluss im Prinzip validierbar sein.

\section{Bezugsrahmen Aalborg-Verpflichtungen}

Die Teilnehmerkommunen haben sich auf die Aalborg-Verpflichtungen als Bezugsrahmen für das strategische Programm geeinigt. In der ersten Projektphase wurden für die zehn Handlungsfelder Bestandsaufnahmen durchgeführt. Nun werden in einem Beteiligungsverfahren quantifizierte Ziele erarbeitet, die im strategischen Programm durch die Gemeinderäte debattiert und verabschiedet werden.

Das strategische Programm ist eine politisch bindende Erklärung der Maßnahmenschwerpunkte für die jeweilige Zeitspanne. Wann immer eine Kommune Maßnahmen durchführt, Projekte umsetzt, Dienstleistungen anbietet, beansprucht dies finanzielle, personelle und natürliche Ressourcen. Ein wichtiger Schritt in Richtung Nachhaltigkeit ist getan, wenn alle drei Ressourcengruppen berücksichtigt werden. Integriertes Ressourcenmanagement nutzt die Sichtweise der Politik, die gewohnt ist, das kommunale Vermögen einzusetzen. Sowohl für natürliche Ressourcen als auch für soziale Gemeinschaftsgüter werden im strategischen Programm Indikatoren und Ziele vereinbart.

\section{Rollen und Verantwortlichkeiten}

Idealerweise beschreiben Indikatoren die Verknüpfung zwischen Bereitstellung von Gemeinschaftsgütern und Verbrauch natürlicher Ressourcen. Solche Indikatoren beschreiben dann die Wirtschaftlichkeit unseres Handelns.

Um den Managementzyklus umzusetzen wird in jeder Teilnehmerkommune zunächst ein schlagkräftiges Projekt- $\rightarrow$ 
team gebildet, dass für die Koordination verantwortlich ist. Dieses wird durch ein Nachhaltigkeitsteam unterstützt, dass idealerweise aus Vertretern aller relevanten Verwaltungsbereiche, der Politik und der beteiligten gesellschaftlichen Gruppen besteht. Das Team hat die Aufgabe, Entscheidungen auf eine breitere Grundlage zu stellen und die Beteiligung der verschiedenen Gruppen am Managementsystem sicherzustellen. Das Bindeglied zwischen Verwaltung und Politik ist der/die Bürgermeister(in). Der Gemeinderat befasst sich zumindest zweimal jährlich mit dem strategischen Programm. Bei der Verabschiedung und bei der Vorlage von Fortschritts- und Abschlussberichten.

\section{Partnerschaftliche Umsetzung}

Nachhaltige Stadtentwicklung ist nicht Sache der Kommunalbehörden allein. Im Gegenteil, die Umsetzung der meisten Maßnahmen zur Verbesserung der Lebensqualität vor Ort kann von Städten und Gemeinden schon aus finanziellen Gründen nur begleitet werden. Sie setzen den Rahmen, den andere Beteiligte - Haushalte, Firmen, Vereine, Nachbargemeinden - in eigener Verantwortung ausfüllen. Die Kommunalbehörden müssen sich hier viel stärker als Partner für die Umsetzung verstehen. Beratend, wo sinnvoll, helfend, wo nötig. Dafür ist eine Plattform nötig sowie auch ,Eigentümerschaft' für die gemeinsame Zielsetzung der nachhaltigen Stadtentwicklung. Im Nachhaltigkeitsmanagement werden zivilgesellschaftliche Akteure deshalb beteiligt, um gemeinsame Ziele zu stecken, Maßnahmen zu planen, ihre Umsetzung zu beobachten und die Ergebnisse zu würdigen. Die Projektstadt Ludwigsburg hat dies erkannt und ihr neues Stadtentwicklungskonzept im Rahmen von Zukunftskonferenzen gemeinsam mit ihren Bürgerinnen und Bürgern erarbeitet. Im Ergebnis wurden elf Zielfelder vereinbart, die nun in so genannten Masterplänen umgesetzt werden. Für jeden Masterplan werden so genannte Prozessverantwortliche benannt - aus Verwaltung und Zivilgesellschaft. Und Ludwigsburg geht noch einen Schritt weiter. In Zukunft soll auch der städtische Haushalt entlang der gemeinsam vereinbarten Zielfelder dargestellt werden.

Es gibt gute Gründe zur Einführung einer integrierten Nachhaltigkeitssteuerung in Kommunen. Vor allem eine verbesserte Leistungs- und Wettbewerbsfähigkeit in einer sich globalisierenden Welt. Wir erleben seit einiger Zeit, dass die überkommenen Wege der Wirtschaftsförderung ausgetreten sind. Es reicht nicht mehr, Grundstücke und Energie zu Schnäppchenpreisen anzubieten. Immer stärker kommt es darauf an, das Gesamtbild eines lebenswerten Umfeldes zu schaffen, in dem gleichzeitig Arbeits- und Wirtschaftsbedingungen als günstig und angenehm empfunden werden. Unternehmende suchen ein verlässliches Umfeld, dass langfristige Planung ermöglicht. Dazu gehören auch qualifizierte Arbeitskräfte, die sich am Arbeits- und Wohnort wohlfühlen. Nur durch die Schaffung einer frischen, lebendigen, schönen Stadt können entsprechende Rahmenbedingungen geschaffen werden. Städte wie Heidelberg haben diesen Weg vor längerer Zeit beschritten. Ihre Bemühungen im gesellschaft- lichen wie im ökologischen Bereich werden mit den besten Wirtschaftsdaten in einem der wirtschaftfreundlichsten Bundesländer belohnt. Unternehmende sind in der Regel bereit, eine solche Stadtentwicklung durch eigene Leistungen zu stützen und mitzutragen. Wichtig ist, dem einen Rahmen in einem kohärenten und nachvollziehbaren Managementsystem zu geben. Viele ermutigende Partnerschaften mit örtlichen Unternehmungen in den Projektkommunen belegen dies.

\section{Interkommunales Lernen}

Die Entwicklung des Nachhaltigkeitsmanagements erfolgt als Schritt-für-Schritt Prozess der beteiligten Städte. Miteinander lernen, von Erfahrungen der anderen profitieren, Lösungen für Fragestellungen bei anderen finden und sich gegenseitig evaluieren sind mithin wichtige Elemente von Managing Urban Europe 25 . In insgesamt vier internationalen Trainings erarbeiten sich die Städte und Gemeinden die Grundlagen für ihr Nachhaltigkeitsmanagement. Begleitet von persönlichen Mentoren setzen sie dies vor Ort um. Unschätzbaren Wert hat das sich bildende Netzwerk von 25 gleichgesinnten Kommunen.

Man könnte meinen, die im Projekt versammelte Streubreite unterschiedlicher Kommunen könnte einem fachlichen und zielführenden Austausch hinderlich sein. Das Gegenteil ist der Fall. Die Millionenstadt Oslo findet nachhaltige Managementlösungen in der 75.000 Einwohner zählenden Stadt Växjö in Schweden. Die deutsche Stadt Neu-Ulm ist Beispiel für eine gelungene Bestandsaufnahme entlang der Aalborg-Verpflichtungen. Und der Berliner Bezirk Lichtenberg wird fündig im kleinen englischen Lewes District Council auf der Suche nach Beispielen gelungener Kriminalprävention. Die Chance liegt in der Vielfalt der Teilnehmer.

\section{Anmerkungen \\ (1) www.mue25.net \\ (2) http://ec.europa.eu/environment/urban/thematic_strategy.htm \\ (3) http://ec.europa.eu/sustainable/sds2005/index_en.htm \\ (4) www.aalborgplus10.dk \\ (5) Siehe Entscheidung des Rates der EU: Dokument 10117/06, Ziffer 29 \\ (6) www.un.org/millenniumgoals/}

I AUTOR + KONTAKT

Holger Robrecht ist Bereichsleiter Nachhaltigkeitsmanagement und Städtisches Regieren am ICLEI - Local Governments for Sustainability. ICLEI ist ein weltweites Städtenetzwerk mit etwa 500 Mitgliedsstädten, -gemeinden und -kreisen, die sich spürbare Verbesserungen der Bedingungen weltweiter Nachhaltigkeit zum Ziel gesetzt haben. ICLEI, Leopoldring 3, 79098 Freiburg. Tel.: 0761/36892-0, E-Mail: holger.robrecht@iclei-europe.org 
(c) 20I0 Authors; licensee IÖW and oekom verlag. This is an article distributed under the terms of the Creative Commons Attribution Non-Commercial No Derivates License (http://creativecommons.org/licenses/by-nc-nd/3.o/), which permits unrestricted use, distribution, and reproduction in any medium, provided the original work is properly cited. 